\title{
Tracking agronomic diversity in land races of cowpea [Vigna unguiculata (L.) Walpers] collected from Pakistan
}

\author{
Muhammad Sajjad Iqbal ${ }^{1}$, Abdul Ghafoor ${ }^{2}$, Sammer Fatima ${ }^{1 *}$, Shamim \\ AKhtar ${ }^{1}$, Muhammad Akbar ${ }^{1}$ and Ejaz Hussain Saddiqi ${ }^{1}$ \\ 1. Department of Botany, University of Gujrat, Gujrat, 50700-Pakistan \\ 2. Plant Genetic Resources Institute, National Agricultural Research Centre, Islamabad-Pakistan \\ *Corresponding author's email: sammer.fatima@uog.edu.pk \\ Citation \\ Muhammad Sajjad Iqbal, Abdul Ghafoor, Sammer Fatima, Shamim AKhtar, Muhammad Akbar and Ejaz Hussain \\ Saddiqi. Tracking agronomic diversity in land races of cowpea [Vigna unguiculata (L.) Walpers] collected from \\ Pakistan. Pure and Applied Biology. Vol. 6, Issue 4, pp1260-1268. http://dx.doi.org/10.19045/bspab.2017.600134
}

\begin{tabular}{llll}
\hline \hline Received: 25/05/2017 & Revised: 20/09/2017 & Accepted: 25/09/2017 & Online First: 27/09/2017
\end{tabular}

\section{Abstract}

Seventy one accessions of Vigna unguiculata (L.) Walpers belonging to 31 districts of three provinces across Pakistan were characterized. High variation was recorded in plant height, branches per plant, pods per plant, grain yield, biomass and harvest index. It has been derived from current studies that migration of germplasm or seeds were observed across various areas that might be due to frequent exchange of grains, between different markets and transportation without any provincial laws implementations. High phenotypic diversity indicates the potential land races collected from farmer's field might be utilized for crop improvement programs viz., 27028, 27029, 27038, 27040, 27042, 27047, 27083 and 27097. Phenotypic diversity analyzed within each district indicated the possibility of migration of landraces at one hand and on the other hand variation appears attributable to different districts without influence that how frequently area was explored. Intra and inter-specific variances deviated from central tendency depicted on the map of Pakistan shall be proved beneficial for future germplasm collectors to devise strategy for collecting genes of interest. New expeditions may be conducted in the main cowpea growing areas with focusing geographically and ecologically distinct areas, rather than fields closer to motorable roads.

Keywords: Underutilized crops; Legumes; Landraces; Genetic diversity

\section{Introduction}

Cowpea (Vigna unguiculata (L.) Walp) is an underutilized crop of tropical Africa, Asia, Central and South America [1, 2]. The crop is well adapted to diverse environmental conditions and can be easily grown in poor soils and semi arid regions [3]. It is rich in protein contents ranging from 22.5 to 25.6 $\%$. Number of vitamins and minerals are present in cowpea that makes it vital for human and animal dietary needs [4-6]. Despite its importance, the production of cowpea ranges from half to one tonne per hectare that is very low as compared to its potential [7]. It is cultivated on marginal soils in Pakistan, especially in NWFP and Northern Punjab on an area of 16.9 thousand hectares with an annual production of 7.8 
thousand metric tonnes [8]. To cater biodiversity impact for socioeconomic values is based on indigenous genetic resources whereas information about geobotanicals is pre-requisite for collecting highest diversity and to devise a fruitful strategy for future collections [9-11].

Intra and inter specific variation played significant role in the improvement of agrobotanical traits. Rate of loss of biodiversity have been studied throughout the world and efforts have been made to preserve the existing biodiversity by means of gene banks, ex-situ conservation at farmer's field, world herbaria and botanical museums by breeders and biologists. During last three decades eighty cowpea accessions have been collected and stored for short duration (5-10 years) at $5^{\circ} \mathrm{C}$, medium term (15-20 years) at $0^{\circ} \mathrm{C}$ and long term (more than 50 years) at $20^{\circ} \mathrm{C}$. These are suggested to be used for evaluation, characterization and planning future strategies for further collections. The cowpea is a neglected crop having potential to become more useful as its genetic diversity is preserved under CBD (Convention on Biological Diversity), whereas major crops like wheat, rice, chickpea and maize have been genetically eroded due to evolution of new cultivars. The aim of the current study was to uncover intra-accession variability occur in collections of 31 districts of three provinces with respect to agronomic traits. It will enable for developing future strategy to preserve high variation with respect to the needs of the growing population.

\section{Materials and methods}

Seventy one (71) landraces, collected predominantly from farmers' fields in 31 districts of three provinces were characterized for agronomic traits to choose high yielding landraces and to make future germplasm collection strategy. During 2013, 71 land races were planted in an augmented design with three replications in the mid of October. Ten single plants from all the accessions were sampled at random, tagged, harvested and threshed individually. Progenies of these individual plants were sown under field conditions during October 2014 and harvested in March 2015. Bed length was maintained at 4 meter, whereas two rows of each accession were planted with 1 meter intra and inter row distance. Plant to plant spacing was kept as $15 \mathrm{~cm}$. After 10 rows, a local check was repeated. Pesticides were sprayed to protect the crop from pests especially white fly (Bemisia tabaci Genn.), a vector for Mungbean Yellow Mosaic Virus (MYMV). Data was recorded following IPGRI descriptors for Vigna unguiculata (IBPGR 1983). Agronomic traits including plant height $(\mathrm{cm})$, branches/plant (number), pods/plant (number), grain yield (g) and biomass yield (g) were recorded. Harvest index was calculated as grain yield over biomass in percentage.

Data recorded were then subjected to statistical analysis by means of variance to find intra and inter-specific variation along with average agronomic performance for individual accessions with deviation from central tendency using the methods provided by Steel and Torrie [12]. Further, variance was derived from summery of statistics and then presented as percent of mean for all characters. Aggregated variances were then calculated for each set of accession, as collections were made from 31 districts. Standard deviation from aggregated variance were taken as an indicator for genetic biodiversity which are represented as in percentage plant samples differed i.e., from none to 100 percent and consequently depicted on the map along with total number of accessions.

\section{Results and discussion}

Average data in 71 accessions collected from 31 districts of Pakistan along with standard error and deviation from means for all the six traits viz., plant height/plant, branches/plant, pods/plant, grain yield/plant, 
biomass/plant and harvest index/plant is presented in Table 1. It is obvious from the results that almost all the characters exhibited a wide inter-accession range which reflecting an idea to choose accessions for high genetic potential. Although variation within accessions was statistically insignificant for most of the accessions but higher magnitude for variation that is reflected in deviation from central point indicated the presence of land races or heterogeneous mixture of homozygous lines (Table 2). Five accessions, 27009, 27029, 27038, 27083 and 27097 gave high yield along with high intraaccession variation that could be evaluated to isolate superior plant progenies within these accessions. Further it could help in identifying plants to be used in hybridization for cowpea improvement.

For strengthening gene pool one way is to collect material from geographically diverse origins from proposed centers of diversity in case of individual samples. Authentic samples from the complete geographical range of the crop species can help maintaining conservation of co-adapted genes [13-15]. Cowpea is an important summer pulse crop and widely cultivated under rainfed areas. Current collection was made from different areas of the country with more emphasis have been provided to those areas which were closer and approachable to motorable roads, that is also indicated in intra accession variance. One of the reasons might be strategy to provide first priority to those areas which are easily approachable and more prominent to genetic erosion and have adaptability to accept advanced breeding material near major towns and cities. Alternatively to gather maximum diversity, it is important to plan and explore those areas which are not explored earlier, preferably like interior territories, remote areas, cross border and mountainous areas.

On the basis of correlation results, pods were observed as the best basic criteria for selection in almost all the collection districts except material from Gujrat, Jhang, Mansehra, Muzzafargarh and Pakpatan (Table 2 ). This might be due to less presentation of area for diversity or uniformity of germplasm collected from these areas. The accessions collected from the districts of Chakwal, Dir, Faisalabad, Jehlum, Lahore, Loralai, Muzzafargarh, Okara, Sheikhupura and Swat were observed low for variance that indicated less representation of these areas although high number of accessions were collected from some of these districts (Figure 1). It could be explained as those maximum accessions might be pure lines with common ancestors. Another threat is exchange of material is frequent within and between various districts which violates seed quarantine laws.

Genetic variation played key role in the determination of genetic diversity (phenotypic \& genotypic) within and between collection sites and it also has expresses an indication for heritable characters potential as reported in brassica [16] barley [17], lentil [18], lupin [19], soybean [20] and cowpea [2, 7]. The variation indicated by the quantitative traits is useful in focusing and designing strategies for exploring and preserving the regions of high genetic diversity. Current findings also support cowpea genetic diversity with respect to the center of genetic diversity [21]. 
lqbal et al.

Table 1. Means along with standard error and deviation for six traits in 71 accessions of cowpea collected from Pakistan

\begin{tabular}{|c|c|c|c|c|c|c|c|c|c|c|c|c|c|}
\hline Accession & Collection site & Plant height & $\sigma$ & Branches/plant & $\sigma$ & Pods/plant & $\sigma$ & Biomass/plant & $\sigma$ & Grain yield & $\sigma$ & Harvest index & $\sigma$ \\
\hline 27008 & Rawalpindi & $142.7 \pm 6.35$ & 20.09 & $7.5 \pm 0.77$ & 2.46 & $25.4 \pm 3.2$ & 10.15 & $33.6 \pm 4.32$ & 13.66 & $11.81 \pm 1.55$ & 4.92 & $35.51 \pm 2.19$ & 6.93 \\
\hline 27009 & Chakwal & $152.3 \pm 6.11$ & 19.33 & $8.7 \pm 0.53$ & 1.70 & $34.3 \pm 5.59$ & 17.69 & $45.95 \pm 9.21$ & 29.14 & $13.83 \pm 2.65$ & 8.39 & $31.24 \pm 2.71$ & 8.58 \\
\hline 27010 & Rawalpindi & $130.0 \pm 9.49$ & 30.04 & $5 \pm 0.59$ & 1.88 & $13.6 \pm 0.93$ & 2.95 & $33.55 \pm 4.97$ & 15.73 & $6.47 \pm 0.39$ & 1.23 & $22.08 \pm 2.74$ & 8.67 \\
\hline 27011 & Rawalpindi & $176.8 \pm 13.25$ & 41.90 & $7 \pm 0.61$ & 1.94 & $24.3 \pm 2.92$ & 9.25 & $45.76 \pm 7.12$ & 22.53 & $9.66 \pm 1.17$ & 3.72 & $23.6 \pm 2.74$ & 8.68 \\
\hline 27012 & Rawalpindi & $143.5 \pm 13.21$ & 41.80 & $5.8 \pm 0.61$ & 1.93 & $20.2 \pm 2.6$ & 8.23 & $35.23 \pm 6.04$ & 19.10 & $11.77 \pm 1.74$ & 5.50 & $34.53 \pm 2.13$ & 6.75 \\
\hline 27018 & Rawalpindi & $153.7 \pm 6.08$ & 19.25 & $7.7 \pm 0.65$ & 2.06 & $38.5 \pm 6.69$ & 21.16 & $41.42 \pm 7.89$ & 24.96 & $12.03 \pm 1.88$ & 5.95 & $29.72 \pm 1.45$ & 4.61 \\
\hline 27022 & Jehlum & $99.6 \pm 6.42$ & 20.32 & $5.1 \pm 0.31$ & 0.99 & $8.3 \pm 1.35$ & 4.30 & $35.1 \pm 4.71$ & 14.90 & $7.36 \pm 1.16$ & 3.67 & $25.81 \pm 4.54$ & 14.36 \\
\hline 27025 & Jehlum & $112.1 \pm 10.72$ & 33.92 & $6.4 \pm 0.47$ & 1.50 & $5.3 \pm 1.39$ & 4.40 & $32.35+5.52$ & 17.47 & $7.18 \pm 1.17$ & 3.72 & $23.44 \pm 3.19$ & 10.09 \\
\hline 27028 & Sialkot & $116.3 \pm 4.69$ & 14.84 & $8 \pm 0.64$ & 2.05 & $23.1+1.82$ & 5.78 & $38.9 \pm 1.79$ & 5.68 & $16.05 \pm 1.5$ & 4.75 & $41.25 \pm 6.67$ & 21.12 \\
\hline 27029 & Sialkot & $131.5 \pm 12.41$ & 39.27 & $9.48 \pm 0.7$ & 2.24 & $29 \pm 4.49$ & 14.22 & $44.74 \pm 3.8$ & 12.03 & $18.21 \pm 2.76$ & 8.74 & $40.68 \pm 14.28$ & 45.16 \\
\hline 27036 & Rawalpindi & $129.1 \pm 9.43$ & 29.84 & $10.5 \pm 0.61$ & 1.96 & $30.1 \pm 2.03$ & 6.42 & $48.03 \pm 2.23$ & 7.06 & $21.44 \pm 1.8$ & 5.70 & $44.63 \pm 5.52$ & 17.49 \\
\hline 27038 & Rawalpindi & $134.0 \pm 15.17$ & 47.99 & $8.9 \pm 0.82$ & 2.60 & $29.5 \pm 5.12$ & 16.22 & $52.85 \pm 3.64$ & 11.51 & $18.72 \pm 2.62$ & 8.32 & $35.42 \pm 23.54$ & 74.47 \\
\hline 27039 & Rawalpindi & $119.0 \pm 9.87$ & 31.21 & $9.6 \pm 0.83$ & 2.63 & $20.5 \pm 2.2$ & 6.98 & $34.62 \pm 1.08$ & 3.44 & $13.04 \pm 1.71$ & 5.42 & $37.66 \pm 13.85$ & 43.80 \\
\hline 27040 & Rawalpindi & $122.1 \pm 10$ & 31.62 & $9.1 \pm 0.67$ & 2.13 & $25 \pm 1.04$ & 3.30 & $46.61 \pm 1.18$ & 3.75 & $18.65 \pm 0.77$ & 2.44 & $40.01 \pm 12.84$ & 40.62 \\
\hline 27041 & Muzaffergarh & $131.2 \pm 4.27$ & 13.52 & $9 \pm 0.64$ & 2.05 & $19.1 \pm 2.07$ & 6.56 & $31.75 \pm 1.98$ & 6.27 & $12.79 \pm 1.16$ & 3.67 & $40.28 \pm 19.35$ & 61.22 \\
\hline 27042 & Rawalpindi & $119.6 \pm 14.11$ & 44.63 & $9.1 \pm 0.72$ & 2.28 & $23.3 \pm 2.07$ & 6.55 & $38.7 \pm 1.89$ & 6.00 & $15.29 \pm 0.92$ & 2.93 & $39.50 \pm 4.38$ & 13.88 \\
\hline 27043 & Lodhran & $98.5 \pm 10.5$ & 33.23 & $9.2 \pm 0.8$ & 2.53 & $20.7 \pm 2.66$ & 8.42 & $45.83 \pm 2.23$ & 7.08 & $14.44 \pm 2.39$ & 7.58 & $25.46 \pm 3.51$ & 80.51 \\
\hline 27044 & Bahawalpur & $131.8 \pm 9.49$ & 30.02 & $11.2 \pm 1.03$ & 3.26 & $19.6 \pm 2.33$ & 7.40 & $27.18 \pm 1.46$ & 4.63 & $12.07 \pm 1.34$ & 4.27 & $44.40 \pm 9.95$ & 31.48 \\
\hline 27045 & Bahawalpur & $121.4 \pm 4.48$ & 14.19 & $9.33 \pm 0.63$ & 2.00 & $20.66 \pm 2.86$ & 9.07 & $43.14 \pm 1.98$ & 6.27 & $13.92 \pm 1.4$ & 4.43 & $32.26 \pm 42.26$ & 133.66 \\
\hline 27047 & Vehari & $138.7 \pm 10.61$ & 33.56 & $7.7 \pm 0.74$ & 2.36 & $39.6 \pm 4.17$ & 13.19 & $58.07 \pm 8.37$ & 26.48 & $16.16 \pm 1.54$ & 4.88 & $29.49 \pm 2.14$ & 6.77 \\
\hline 27052 & Chakwal & $121.7 \pm 7.64$ & 24.18 & $6.55 \pm 0.51$ & 1.64 & $33.55 \pm 7.45$ & 23.58 & $75.11 \pm 11.57$ & 36.62 & $10.11 \pm 2.3$ & 7.30 & $12.81 \pm 1.82$ & 5.76 \\
\hline 27064 & Islamabad & $91.4 \pm 9.91$ & 31.35 & $6.66+0.51$ & 1.63 & $7.22 \pm 1.78$ & 5.65 & $44.97 \pm 8.62$ & 27.28 & $1.25 \pm 0.31$ & 0.99 & $4.06+1.58$ & 5.00 \\
\hline 27075 & Lahore & $111.8 \pm 6.79$ & 21.50 & $5.33 \pm 0.47$ & 1.49 & $6 \pm 2.25$ & 7.12 & $46.33 \pm 7.82$ & 24.74 & $2.94 \pm 1.19$ & 3.77 & $5.53 \pm 1.43$ & 4.53 \\
\hline 27076 & Lahore & $106.0 \pm 8.13$ & 25.72 & $4.88 \pm 0.37$ & 1.20 & $11.55 \pm 1.66$ & 5.27 & $12.13 \pm 1.9$ & 6.02 & $3.35 \pm 0.42$ & 1.36 & $30.8 \pm 3.92$ & 12.41 \\
\hline 27077 & Lahore & $134.4 \pm 12.93$ & 40.90 & $7.4 \pm 0.4$ & 1.26 & $20.3 \pm 6.1$ & 19.30 & $62.43 \pm 9.12$ & 28.84 & $4.83 \pm 1.43$ & 4.53 & $8.63 \pm 3.08$ & 9.74 \\
\hline 27078 & Kasur & $82.0 \pm 5.78$ & 18.29 & $3.7 \pm 0.3$ & 0.95 & $7.2 \pm 1.94$ & 6.16 & $7.37 \pm 1.91$ & 6.05 & $1.5 \pm 0.56$ & 1.78 & $18.7 \pm 2.75$ & 8.72 \\
\hline 27081 & Kasur & $118.1 \pm 6.97$ & 22.07 & $7.2 \pm 0.46$ & 1.47 & $18.6 \pm 3.63$ & 11.48 & $70.69 \pm 10.69$ & 33.80 & $7.22 \pm 1.38$ & 4.38 & $10.18 \pm 1.05$ & 3.33 \\
\hline 27082 & Kasur & $78.2 \pm 6.3$ & 19.95 & $6 \pm 0.51$ & 1.63 & $15.9 \pm 3.1$ & 9.83 & $13.36 \pm 2.23$ & 7.08 & $3.78 \pm 0.92$ & 2.99 & $30.17 \pm 4.43$ & 14.03 \\
\hline 27083 & Gujranwala & $113.4 \pm 7.83$ & 24.78 & $7.8 \pm 0.57$ & 1.81 & $42.8 \pm 3.99$ & 12.63 & $50.51 \pm 5.03$ & 15.94 & $18.17 \pm 3.05$ & 9.64 & $35.7 \pm 4.07$ & 12.88 \\
\hline 27084 & Sahiwal & $114.7 \pm 10.39$ & 32.88 & $8 \pm 0.91$ & 2.91 & $19.2 \pm 3.74$ & 11.86 & $15.09 \pm 3.77$ & 11.94 & $6.12 \pm 1.56$ & 4.94 & $40.21 \pm 1.31$ & 4.14 \\
\hline 27087 & Khushab & $100.6 \pm 9$ & 28.48 & $7.5 \pm 0.77$ & 2.46 & $12.7 \pm 1.25$ & 3.97 & $10.98 \pm 1.26$ & 4.01 & $3.67 \pm 0.43$ & 1.36 & $33.36 \pm 1.7$ & 5.38 \\
\hline 27088 & Okara & $97.3 \pm 15.67$ & 49.58 & $4.9 \pm 0.92$ & 2.92 & $21.2 \pm 4.05$ & 12.81 & $32.1 \pm 6.31$ & 19.97 & $8.89 \pm 1.84$ & 5.82 & $27.89 \pm 3.71$ & 11.74 \\
\hline 27089 & Gujranwala & $104.5 \pm 2.87$ & 9.09 & $7.6 \pm 0.33$ & 1.07 & $16.3 \pm 2.45$ & 7.76 & $37.05 \pm 2.86$ & 9.07 & $13.19 \pm 2.14$ & 6.78 & $35.60 \pm 21.30$ & 67.36 \\
\hline 27091 & Gujrat & $101.7 \pm 9.73$ & 30.78 & $7.6 \pm 0.63$ & 2.01 & $19.3 \pm 2.99$ & 9.46 & $47.84 \pm 2.32$ & 7.34 & $14.07 \pm 1.49$ & 4.73 & $29.41 \pm 16.66$ & 52.68 \\
\hline 27093 & Haripur & $107.9 \pm 8.58$ & 27.15 & $6 \pm 0.61$ & 1.94 & $16.2 \pm 3.05$ & 9.65 & $36.47 \pm 7.16$ & 22.66 & $6.63 \pm 0.87$ & 2.76 & $20.75 \pm 2.97$ & 9.40 \\
\hline 27094 & Quetta & $93.5 \pm 5.31$ & 16.80 & $6 \pm 0.66$ & 2.11 & $14.8 \pm 2.69$ & 8.51 & $17.46 \pm 2.36$ & 7.48 & $5.75 \pm 1.09$ & 3.46 & $32.58 \pm 3.75$ & 11.88 \\
\hline
\end{tabular}




\begin{tabular}{|c|c|c|c|c|c|c|c|c|c|c|c|c|c|}
\hline 27095 & Loralai & $138.5 \pm 3.57$ & 11.30 & $6.18 \pm 0.51$ & 1.62 & $10.61 \pm 2.03$ & 6.45 & $35.86 \pm 6.61$ & 20.93 & $5.5 \pm 1.03$ & 3.26 & $18.25 \pm 3.86$ & 12.22 \\
\hline 27096 & Chitral & $74.0 \pm 9.35$ & 29.59 & $6.1 \pm 0.48$ & 1.52 & $18.4 \pm 3.98$ & 12.62 & $37.82 \pm 6.81$ & 21.55 & $12.03 \pm 3.13$ & 9.92 & $30.57 \pm 3.45$ & 10.92 \\
\hline 27097 & Chitral & $114.2 \pm 13.09$ & 41.42 & $5.4 \pm 0.45$ & 1.43 & $17.9 \pm 3.19$ & 10.09 & $37.38 \pm 5.2$ & 16.46 & $16.25 \pm 8.32$ & 26.32 & $36.42 \pm 11.64$ & 36.82 \\
\hline 27098 & Chitral & $92.2 \pm 5.7$ & 18.04 & $8.2 \pm 0.99$ & 3.15 & $17.9 \pm 4.01$ & 12.71 & $49.01 \pm 9.53$ & 30.17 & $8.71 \pm 1.54$ & 4.88 & $18.99 \pm 1.72$ & 5.46 \\
\hline 27099 & Sheikhupura & $108.0 \pm 7.24$ & 22.90 & $6.75 \pm 0.44$ & 1.39 & $10.5 \pm 1.86$ & 5.91 & $65.82 \pm 12.73$ & 40.27 & $9.72 \pm 1.35$ & 4.28 & $16.2 \pm 1.45$ & 4.59 \\
\hline 27102 & Gilgit & $107.1 \pm 10.86$ & 34.36 & $7.78 \pm 0.44$ & 1.40 & $4.66 \pm 0.85$ & 2.71 & $23.75 \pm 4.54$ & 14.38 & $1.78 \pm 0.42$ & 1.36 & $7.97 \pm 1.71$ & 5.44 \\
\hline 27106 & Layyah & $143.0 \pm 10.85$ & 34.32 & $7.1 \pm 0.5$ & 1.59 & $3.24 \pm 0.72$ & 2.30 & $34.44 \pm 2.36$ & 7.47 & $3.82 \pm 0.65$ & 2.05 & $10.84 \pm 1.2$ & 3.80 \\
\hline 27107 & Bahawalpur & $129.0 \pm 7.04$ & 22.28 & $7.1 \pm 0.82$ & 2.60 & $50.1 \pm 8.75$ & 27.67 & $69.42 \pm 12.95$ & 40.95 & $18.8 \pm 2.9$ & 9.19 & $29.18 \pm 2.08$ & 6.60 \\
\hline 27115 & Mansehra & $145.0 \pm 11.95$ & 37.80 & $8.4 \pm 0.85$ & 2.71 & $13.9 \pm 4.01$ & 12.70 & $55.08 \pm 8$ & 25.30 & $5.74 \pm 1.86$ & 5.89 & $9.79 \pm 1.73$ & 5.49 \\
\hline 27116 & Swat & $129.2 \pm 10.89$ & 34.45 & $6.9 \pm 0.58$ & 1.85 & $17.5 \pm 3.42$ & 10.83 & $52.48+4.78$ & 15.13 & $6.83 \pm 1.36$ & 4.31 & $12.63+2.13$ & 6.75 \\
\hline 27118 & Swat & $115.8 \pm 7.18$ & 22.71 & $8 \pm 0.74$ & 2.36 & $14.2 \pm 1.45$ & 4.59 & $37.74 \pm 2.98$ & 9.43 & $6.29 \pm 0.81$ & 2.58 & $17.81 \pm 2.73$ & 8.66 \\
\hline 27119 & Mansehra & $110.4 \pm 5.07$ & 16.03 & $5.96 \pm 0.61$ & 1.95 & $8.62 \pm 1.16$ & 3.68 & $57.85 \pm 6.24$ & 19.76 & $10.12 \pm 1.58$ & 5.03 & $17.5 \pm 2.63$ & 8.32 \\
\hline 27120 & Mansehra & $111.2 \pm 5.71$ & 18.07 & $8.2 \pm 0.87$ & 2.78 & $6.5 \pm 1.96$ & 6.20 & $35.72 \pm 4.96$ & 15.71 & $1.86 \pm 0.69$ & 2.20 & $5.81 \pm 2.33$ & 7.39 \\
\hline 27121 & Dir & $105.5 \pm 6.86$ & 21.71 & $7.4 \pm 0.68$ & 2.17 & $14.7 \pm 3.11$ & 9.85 & $42.93 \pm 3.69$ & 11.69 & $4.19 \pm 0.87$ & 2.75 & $10.71 \pm 2.26$ & 7.16 \\
\hline 27122 & Dir & $99.6 \pm 3.62$ & 11.45 & $6.6 \pm 0.56$ & 1.77 & $7.9 \pm 0.95$ & 3.03 & $24.19 \pm 5.15$ & 16.30 & $5.91 \pm 1.88$ & 5.94 & $29.7 \pm 7.71$ & 24.40 \\
\hline 27123 & Dir & $129.5 \pm 9.25$ & 29.23 & $7.5 \pm 0.74$ & 2.37 & $9.5 \pm 2.01$ & 6.36 & $31.8 \pm 6.12$ & 19.36 & $5.65 \pm 1.98$ & 6.27 & $16.49 \pm 2.69$ & 8.53 \\
\hline 27124 & Dir & $95.0 \pm 8.36$ & 26.44 & $5.4 \pm 0.45$ & 1.43 & $8.3 \pm 2.29$ & 7.26 & $19.27 \pm 2.94$ & 9.31 & $6.38 \pm 2.83$ & 8.97 & $33.42 \pm 14.83$ & 46.92 \\
\hline 27125 & Chitral & $119.0 \pm 5.83$ & 18.45 & $6.9 \pm 0.65$ & 2.08 & $8.9 \pm 1.66$ & 5.28 & $30.76 \pm 5.68$ & 17.97 & $3.64 \pm 0.84$ & 2.68 & $12.83 \pm 3.31$ & 10.48 \\
\hline 27126 & Chitral & $106.0 \pm 7.99$ & 25.29 & $6.53 \pm 0.3$ & 0.95 & $7.91 \pm 2.03$ & 6.43 & $21.08 \pm 2.71$ & 8.59 & $2.75 \pm 0.79$ & 2.52 & $10.82 \pm 2.13$ & 6.75 \\
\hline 27127 & Chitral & $116.0 \pm 5.32$ & 16.84 & $5.8 \pm 0.57$ & 1.81 & $12.4 \pm 3.24$ & 10.25 & $27.98 \pm 4.56$ & 14.43 & $5.87 \pm 1.25$ & 3.97 & $23.96 \pm 5.1$ & 16.13 \\
\hline 27140 & Dir & $133.6 \pm 11.15$ & 35.26 & $9.2 \pm 0.89$ & 2.82 & $24.7 \pm 2.97$ & 9.39 & $58.18 \pm 7.15$ & 22.64 & $11.66 \pm 1.69$ & 5.35 & $20.89 \pm 3.07$ & 9.71 \\
\hline 27142 & Multan & $102.9 \pm 14.77$ & 46.72 & $9.54 \pm 0.75$ & 2.40 & $22.5 \pm 1.74$ & 5.50 & $20.91 \pm 2.47$ & 7.83 & $4.78 \pm 1.06$ & 3.36 & $27.02 \pm 7.33$ & 23.20 \\
\hline 27143 & Bahawalpur & $127.1 \pm 10.91$ & 34.52 & $9.2 \pm 0.98$ & 3.12 & $19.6 \pm 2.82$ & 8.95 & $20.63 \pm 3.11$ & 9.84 & $4.34 \pm 0.59$ & 1.88 & $28.25 \pm 6.89$ & 21.80 \\
\hline 27144 & Lahore & $145.2 \pm 10.68$ & 33.79 & $9.2 \pm 0.62$ & 1.99 & $26.5 \pm 3.11$ & 9.83 & $26.39 \pm 3.87$ & 12.25 & $6.59 \pm 1.14$ & 3.63 & $25.5 \pm 2.42$ & 7.66 \\
\hline 27145 & Gujranwala & $125.1 \pm 8.17$ & 25.86 & $8.1 \pm 0.69$ & 2.18 & $17.9 \pm 2.4$ & 7.59 & $25.05 \pm 1.75$ & 5.55 & $7.22 \pm 1.14$ & 3.63 & $28.82 \pm 15.8$ & 49.98 \\
\hline 27149 & Dir & $121.6 \pm 11.13$ & 35.20 & $10.25 \pm 0.99$ & 3.16 & $14.14 \pm 4.14$ & 13.11 & $44.31 \pm 4.06$ & 12.85 & $3.18 \pm 0.92$ & 2.93 & $7.08 \pm 1.66$ & 5.27 \\
\hline 27151 & Dir & $116.7 \pm 5.46$ & 17.27 & $5.2 \pm 0.46$ & 1.47 & $21.6 \pm 1.93$ & 6.13 & $24.18 \pm 2.47$ & 7.82 & $6.28 \pm 1.06$ & 3.38 & $27.37 \pm 4.77$ & 15.11 \\
\hline 27154 & Jhang & $149.2 \pm 9.39$ & 29.72 & $11.62 \pm 0.76$ & 2.42 & $7.72 \pm 1.57$ & 4.97 & $37.25 \pm 4.34$ & 13.75 & $2.83 \pm 0.56$ & 1.78 & $7.81 \pm 1.09$ & 3.45 \\
\hline 27158 & Faisalabad & $99.0 \pm 8.15$ & 25.80 & $5.11 \pm 0.37$ & 1.20 & $17.88 \pm 2.14$ & 6.77 & $17.14 \pm 1.99$ & 6.32 & $5.41 \pm 0.68$ & 2.16 & $31.58 \pm 2.21$ & 7.00 \\
\hline 27160 & Okara & $118.0 \pm 8.93$ & 28.25 & $5.9 \pm 0.72$ & 2.28 & $17.9 \pm 1.71$ & 5.42 & $23.99 \pm 3.29$ & 10.41 & $6.61 \pm 1.15$ & 3.64 & $29.43 \pm 4.13$ & 13.09 \\
\hline 27161 & Pakpattan & $103.9 \pm 1.17$ & 3.72 & $5.43 \pm 0.14$ & 0.45 & $25.96 \pm 0.53$ & 1.69 & $29.94 \pm 2.46$ & 7.78 & $8.91 \pm 0.62$ & 1.97 & $30.64 \pm 1.75$ & 5.55 \\
\hline 27170 & Dir & $121.6 \pm 16.68$ & 52.77 & $9.38 \pm 0.57$ & 1.82 & $15.96 \pm 3.45$ & 10.92 & $48.86 \pm 6.66$ & 21.06 & $2.01 \pm 0.57$ & 1.80 & $3.84 \pm 0.81$ & 2.58 \\
\hline 27171 & Chitral & $103.0 \pm 13.96$ & 44.15 & $7.94 \pm 0.72$ & 2.31 & $9.78 \pm 3.11$ & 9.84 & $44.54 \pm 7.73$ & 24.46 & $2.2 \pm 0.49$ & 1.55 & $6.73 \pm 1.89$ & 6.00 \\
\hline 27172 & Chitral & $103.8 \pm 7.57$ & 23.95 & $7.8 \pm 1.07$ & 3.39 & $8.1 \pm 1.2$ & 3.81 & $64.42 \pm 12.98$ & 41.07 & $1.8 \pm 0.24$ & 0.78 & $6.12 \pm 2.29$ & 7.27 \\
\hline Check & Narowal & $109.9 \pm 8.95$ & 28.31 & $8.2 \pm 0.66$ & 2.10 & $27.1 \pm 2.21$ & 7.01 & $98.46 \pm 14.53$ & 45.97 & $5.88 \pm 1.19$ & 3.77 & $9.99 \pm 4.68$ & 14.82 \\
\hline $\mathrm{CV}(\%)$ & & 15.17 & & 17.86 & & 14.98 & & 24.46 & & 16.28 & & 11.12 & \\
\hline
\end{tabular}


Table 2. Simple correlation of grain yield with other five agronomic traits for cowpea germplasm collected from Pakistan

\begin{tabular}{|c|c|c|c|c|c|c|}
\hline District & Frequency & Plant height & $\begin{array}{l}\text { Branches } \\
\text { per plant }\end{array}$ & $\begin{array}{c}\text { Pods per } \\
\text { plant }\end{array}$ & $\begin{array}{c}\text { Biomass per } \\
\text { plant }\end{array}$ & Harvest index \\
\hline Bahawalpur & 40 & 0.06 & 0.10 & $0.76^{* * *}$ & $0.67^{\text {** }}$ & 0.31 \\
\hline Chakwal & 20 & 0.10 & 0.40 & $0.90^{* * *}$ & $0.66^{\text {*** }}$ & 0.37 \\
\hline Chitral & 80 & 0.11 & -0.06 & $0.66^{* *}$ & 0.09 & $0.89^{* *}$ \\
\hline Dir & 80 & 0.05 & 0.11 & $0.36^{* * *}$ & $0.34^{* * *}$ & $0.68^{* *}$ \\
\hline Faisalabad & 10 & $0.72^{*}$ & 0.05 & $0.74^{*}$ & $0.85^{* *}$ & 0.50 \\
\hline Gilgit & 10 & 0.11 & $0.69^{*}$ & $0.74^{*}$ & $0.75^{*}$ & 0.53 \\
\hline Gujranwala & 30 & $-0.52^{* *}$ & $0.41^{*}$ & $0.82^{* * *}$ & 0.21 & 0.25 \\
\hline Gujrat & 10 & -0.15 & -0.25 & -0.21 & -0.11 & $0.84^{* *}$ \\
\hline Haripur & 10 & -0.08 & 0.35 & $0.79^{* *}$ & $0.65^{*}$ & 0.20 \\
\hline Islamabad & 10 & -0.13 & 0.34 & $0.97^{* * *}$ & 0.28 & $0.87^{* *}$ \\
\hline Jehlum & 20 & 0.16 & $0.56^{* *}$ & $0.85^{* * *}$ & $0.82^{* * *}$ & 0.06 \\
\hline Jhang & 10 & 0.04 & -0.14 & 0.08 & 0.09 & $0.70^{*}$ \\
\hline Kasur & 30 & 0.18 & $0.39^{*}$ & $0.43^{*}$ & $0.90^{\text {** }}$ & $0.48^{* *}$ \\
\hline Khushab & 10 & 0.11 & 0.51 & $0.67^{*}$ & 0.32 & 0.41 \\
\hline Lahore & 40 & -0.27 & $0.32^{*}$ & $0.91^{* * *}$ & $0.65^{\text {** }}$ & $0.94^{* * *}$ \\
\hline Layyah & 10 & -0.08 & 0.15 & $0.97^{* *}$ & 0.07 & $0.66^{*}$ \\
\hline Lodhran & 10 & -0.29 & 0.17 & $0.64^{*}$ & $0.69^{*}$ & $0.78^{* * *}$ \\
\hline Loralai & 10 & 0.54 & -0.12 & $0.91^{\text {*** }}$ & 0.01 & $0.86^{* *}$ \\
\hline Mansehra & 30 & 0.14 & 0.29 & -0.25 & $-0.40^{*}$ & $0.94^{* *}$ \\
\hline Multan & 10 & 0.01 & 0.28 & $0.73^{*}$ & 0.52 & 0.12 \\
\hline Muzzafargarh & 10 & 0.20 & 0.33 & 0.11 & -0.07 & 0.66 \\
\hline Okara & 20 & 0.15 & 0.38 & $0.58^{* *}$ & $0.86^{\text {** }}$ & 0.33 \\
\hline Pakpattan & 10 & 0.00 & $-0.87^{* *}$ & $-0.59^{* *}$ & $0.74^{*}$ & 0.25 \\
\hline Quetta & 10 & 0.36 & 0.60 & $0.94^{* * *}$ & $0.83^{* *}$ & 0.60 \\
\hline Rawalpindi & 100 & 0.01 & $0.53^{* *}$ & $0.83^{* * *}$ & $0.65^{\text {** }}$ & $0.34^{* * *}$ \\
\hline Sahiwal & 10 & 0.46 & $0.82^{* * *}$ & $0.82^{* * *}$ & $0.94^{* * *}$ & 0.20 \\
\hline Sheikhupura & 10 & 0.46 & $0.78^{* * *}$ & $0.91^{\text {*** }}$ & $0.91^{\text {*** }}$ & -0.25 \\
\hline Sialkot & 20 & $0.71^{\text {*** }}$ & $0.69^{* * *}$ & $0.94^{* * *}$ & $0.58^{\text {*** }}$ & $0.47^{*}$ \\
\hline Swat & 20 & -0.20 & -0.10 & $0.91^{* * *}$ & -0.17 & $0.87^{* *}$ \\
\hline Vehari & 10 & 0.07 & 0.43 & $0.95^{* *}$ & $0.84^{* * *}$ & -0.10 \\
\hline
\end{tabular}

*Significant and ${ }^{* *}$ highly significant, respectively

Eight accessions, i.e., 27028, 27029, 27038, 27040, 27042, 27047, 27083 and 27097 were found as high grain yielding and could be used for crop improvement programs. Van Hintum and Elings reported that rare alleles are also functional as mutants or elements of migratory populations which may be results of various events [22]. In the countries like Pakistan there is no restriction for seed material from one area to other, due to it migration of landraces may not be pure lots and chances of contamination are high. The area with high stress may have the ability to show tolerance against abiotic and biotic stresses but mixing posses serious threat to sampling of genetic resources from that area. Whereas pure lots or sampling at right time with appropriate conditions may prove helpful for collecting such materials using them in the future hybrid development programs.

Supporting the view in another study, Laghetti considered oppressive prolonged drought a serious threat to the conservation of gene pool of Vigna savi in natural habitat and thus recommended germplasm collection mission for conservation of maximum genetic diversity from the areas under environmental stresses [23]. Although cowpea is being cultivated in the Province 
of Sindh but no representation is available in the gene bank that is needed to explore this area prior to genetic erosion due to introduction of superior cultivars of cowpea or other alternative crops. This material is expected to provide some good lines tolerant to drought.

Additionally it is suggested from current findings that areas with rich biodiversity of cowpea should be explored systematically and efforts should also be concentrated on geographically and ecologically distinct areas as possible, rather approachable sites only. Remote areas and provinces which have more genetic resources to focus required number of expeditions and collection missions with the involvement of local community who are the ultimate stake holders as also been advocated for tetraploid wheat $[24,25]$. Further building of genetic stocks required expeditions of those areas where genetic erosion is expected like in cowpea along with the locations from where existing genetic diversity has not yet gathered is recommended to explore for genepool establishment and starting successful breeding program [26]. Current work could help in devising future strategy for cowpea germplasm collections across the country.

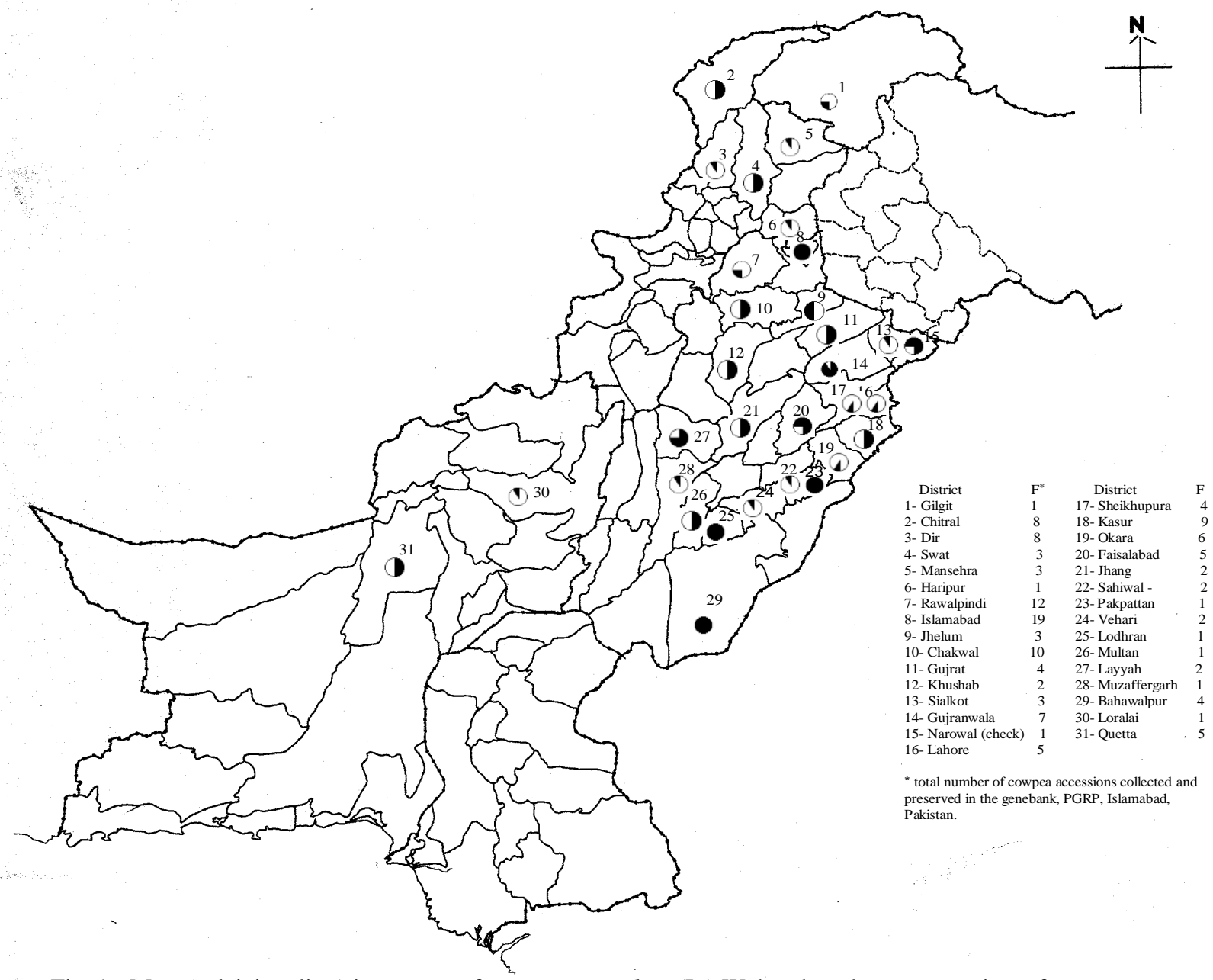

Fig. 1:- Map explaining diversity pattern of Vigna unguiculata (L.) Walper based on agronomic performance. The symbols represent as, $\bigcirc$ - Insignificant differences, $\bigcirc<25 \%$ plants differed, $>50 \%$ plants differed, - $<5 \%$ plants differed and $-100 \%$ plant samples differed for inter and intra-accession performance. 


\section{Conclusion}

Cowpea is a protein rich food crop which is recommended for not only marginal lands but for areas with low irrigation. There is need to conserve and preserve cowpea genetic resources on comprehensive basis before they might be lost due to various environmental factors. Small scale crop specific genetic resources conservation station is also suggested to be establish in the remote areas to save unique genes of interest.

\section{Authors' contributions}

Conceived and designed the experiments: A Ghafoor, Performed the experiments: MS Iqbal, Analyzed the data: MS Iqbal \& A Ghafoor, Contributed materials/ analysis/ tools: S Fatima, S Akhtar, M Akbar \& EH Saddiqi Wrote the paper: MS Iqbal \& A Ghafoor.

\section{References}

1. Iqbal MS, Ghafoor A, Qureshi AS \& Ahmad Z (2003). Genetic dissimilarities in Cowpea (Vigna unguiculata (L.) Walp.) for protein peptides and their significance for quantitative traits loci. Pak J Bot 35(3): 377-386.

2. Coulibaly S, Pasquet RS, Papa R \& Gepts $P$ (2002). AFLP analysis of the phenetic organization and genetic diversity of Vigna unguiculata L. Walp., reveals extensive gene flow between wild and domesticated types. Theor Appl Genet 104: 358-366.

3. Boye J, Zare F \& Pletch A (2010). Pulse proteins: processing, characterization, functional properties and applications in food and feed. Food Res Int 43: 414-43.

4. Rachie KO (1985). Introduction in: eds. S. R. Singh and K. O. Rachie, Cowpea Researches, Production and Utilization. John Wiley, New York.

5. Nielsen S, Ohler T \& Mitchell C (1997). Cowpea leaves for human consumption: production, utilization, and nutrient composition. In: Singh B, D. Mohan Raj, K. Dashiell and L. Jackai (eds.) advances in cowpea research. IITA-JIRCAS, Ibadan, Nigeria. 326-332.

6. Antova GA, Stoilova TD, Ivanova MM (2014). Proximate and lipid composition of cowpea (Vigna unguiculata L.) cultivated in Bulgaria. J Food Comp Anal 33:146-152.

7. Fall L, Diouf D, Fall-Ndiaye MA, Badiane FA \& Gueye M (2003). Genetic diversity in cowpea (Vigna unguiculata (L.) Walp.) varieties determined by ARS and RAPD techniques. Afr $J$ Biotech 2(2): 48-50.

8. Iqbal MS, Qureshi AS, Ghafoor A and Qayyum A (2003). Identification of superior genotypes based on morphological, physiological and agronomic traits in local and exotic cowpea germplasm. Pak J Bot 35(1): 6977.

9. Bashir M (1992). Serological and biological characterization of seed borne isolates of blackeye cowpea mosaic and cowpea aphid-borne mosaic potyviruses in Vigna unguiculata (L.) Walp. PhD thesis, Oregon State University, OR, USA. 222 pp.

10. Iqbal MS, Qureshi AS \& Ghafoor A (2010). Evaluation of Nigella sativa L. for genetic variation and ex-situ conservation. Pak J Bot 42(4): 24892495.

11. Ghafoor A, Gill MA, Hassan A, Murtaza G \& Qadir M (2001). Gypsum: An economical amendment for amelioration of saline-sodic waters and soils for improving crop yields. Int J Agri Biol 3: 266-275.

12. Steel RGD \& Torrie JH (1980). Principles and procedures of statistics. A Biometrical Approach, $2^{\text {nd }}$ Ed. McGrawHill, New York. 
13. Omoigui LO, Ekeuro GC, Kamara AY, Bello LL, Timko MP, \& Ogunwolu GO (2017). New sources of aphids [Aphis craccivora (Koch)] resistance in cowpea germplasm using phenotypic and molecular marker approaches. Euphytica 213(8): 178.

14. Omoigui LO, Kamara AY, Alunyo GI, Bello LL, Oluoch M, Timko MP \& Boukar O. (2017). Identification of new sources of resistance to Striga gesnerioides in cowpea Vigna unguiculata accessions. Genet Resour Crop Evo 64(5): 901-911.

15. Tani E, Abraham E, Chachalis D \& Travlos I (2017). Molecular, genetic and agronomic approaches to utilizing pulses as cover crops and green manure into cropping systems. Int J Mol Sci 18(6): 1202.

16. Zhang W, Hu D, Raman R, Guo S, Wei Z, Shen X, Meng J, Raman H \& Zou J (2017). Investigation of the genetic diversity and quantitative trait loci accounting for important agronomic and seed quality traits in Brassica carinata. Front Plant Sci 8: 615.

17. Allel D, Ben-Amar A, Lamine $M$ \& Abdelly C (2017). Relationships and genetic structure of North African barley (Hordeum vulgare L.) germplasm revealed by morphological and molecular markers: Biogeographical considerations. S Afr J Bot 112: 1-10.

18. Tahir NAR \& Omer DA (2017). Genetic variation in lentil genotypes by morphoagronomic traits and RAPD-PCR. $J$ Anim Plant Sci 27(2): 468-480.

19. Clements JC \& Cowling WA (1994). Pattern of morphological diversity in relation to geographical origins of wild Lupinus angustifolius from the Aegean region. Genet Resour Crop Evo, 41: 109122.

20. Oliveira MM, Sousa LB, Reis MC, Silva JE, Cardoso DBO, Hamawaki OT \& Nogueira APO (2017). Evaluation of genetic diversity among soybean (Glycine max) genotypes using univariate and multivariate analysis. Genet Mol Res 16(2): 1-10.

21. Menssen M, Linde M, Omondi EO, Abukutsa-Onyango M, Dinssa FF \& Winkelmann T (2017). Genetic and morphological diversity of cowpea (Vigna unguiculata (L.) Walp.) entries from East Africa. Sci Hort 226: 268-276.

22. Van Hintum TJL \& Elings A (1991). Assessment of glutenin and phenotypic diversity of Syrian durum wheat landraces in relation to their geographical origin. Euphytica, 55: 209-215.

23. Laghetti G, Pienaar BL, Padulosi S \& Perrino P (1998). Ecogeographical distribution of Vigna savi in Southern Africa and some areas of the Mediterranean basin. Plant Genet Resour News 115: 6-12.

24. Pecetti L, Annicchiario P \& Damania AB (1992). Biodiversity in a germplasm collection of durum wheat. Euphytica 60: 229-238.

25. Pecetti L, Annicchiario P \& Damania AB (1996). Geographic variation in tetraploid wheat (Triticum turgidum spp. Turgidum convar. Durum) landraces from two provinces in Ethiopia. Euphytica, 43: 395-407.

26. Padulosi S (1993). Genetic diversity, taxonomy and ecogeographic survey of the wild relatives of cowpea (Vigna unguiculata L. Walp.). Ph. D. Thesis, Catholic University of Louvain-LaNeuve, Belgium. 